\title{
Post partum headache: a case series
}

\section{Reema Kumar Bhatt*, Rohin Kumar, L. B. Singh}

Department of Obstetrics and Gynecology, Army Hospital Research and Referral, Delhi, India

Received: 13 November 2018

Accepted: 03 December 2018

\section{*Correspondence:}

Dr. Reema Kumar Bhatt,

E-mail: reemakamalbhatt@yahoo.co.in

Copyright: (c) the author(s), publisher and licensee Medip Academy. This is an open-access article distributed under the terms of the Creative Commons Attribution Non-Commercial License, which permits unrestricted non-commercial use, distribution, and reproduction in any medium, provided the original work is properly cited.

\begin{abstract}
Postpartum headache is the complaint of head, neck, or shoulder pain occurring during the first 6 weeks following delivery. Among the women who underwent neuraxial aneasthesia Post Dural puncture headache is one of the most common presentations. However, physicians should be aware that post Dural puncture headache is not the only cause of postpartum headache. Authors present a series of four cases that had varied presentation of post partum headache with varied diagnosis, the first case was of subdural haematoma where CT scan revealed an acute on chronic SDH. The second case was diagnosed as meningitis and the CSF for culture sensitivity grew Streptococcus Pneumoniae .In our third case of cortical vein thrombosis, NCCT followed by MR venogram revealed left transverse venous sinus thrombosis and our last case in the series was of posterior reversible encephalopathy syndrome where MRI confirmed the findings of typical findings are symmetric edema involving the white matter of the posterior regions of the cerebral hemispheres. The diagnostic dilemma was resolved by neuroimaging because the clinical presentation was not making us reach a satisfactory diagnosis of the cause of headache. Early resort to neuroimaging and multidisciplinary team effort paved way to early diagnosis and appropriate recovery of the patients.
\end{abstract}

Keywords: Cortical vein thrombosis, Meningitis, Multidisciplinary management, Neuroimaging, Posterior reversible encephalopathy syndrome, Postpartum headache, Subdural hematoma

\section{INTRODUCTION}

Headache is a common symptom which a postpartum lady presents in the OPD. It is generally attributed to lack of sleep, fatigue from looking after the baby and sinister causes are generally ignored. However, the symptom of headache in postpartum period requires consideration and evaluation. Headache is classified into primary and secondary head-ache according to international classification of headache disorders (ICHD II).

Primary headache include migraine, cluster headache and tension headache. Secondary head-ache is due to an underlying pathology. Primary headache is 20 times more common than secondary headache in postpartum period. Tension headache, migraine, musculo-skeletal, post dural puncture headache and headache due to preeclampsia and eclampsia constitute major causes of postpartum headache.

Other causes which include head-ache due to sub arachnoid haemorrhage, posterior reversible encephalopathy syndrome, subdural hematoma, meningitis and cerebral venous sinus thrombosis need to be ruled out when evaluating a patient for postpartum headache. ${ }^{1,2}$

\section{CASE REPORT}

Authors present a case series of Post-partum headache with varied diagnosis and how they were managed at our hospital. 


\section{Case 1}

36 yrs old primipara who underwent emergency LSCS at POG 36 weeks 06 days on for Post IVF ET twin pregnancy with first breech in labour with pre-eclampsia. In the post-operative blood pressure measurements were within normal limits and patient was discharged 03 days after surgery.

She was readmitted 19 days after discharge with complaints of left sided headache associated with left sided blurring of vision. There was no history of fever, vomiting and during examination revealed no neurological deficit. CT scan revealed an acute on chronic SDH left frontotemporo parietal region with midline shift of $7 \mathrm{~mm}$ as shown (Figure 1).

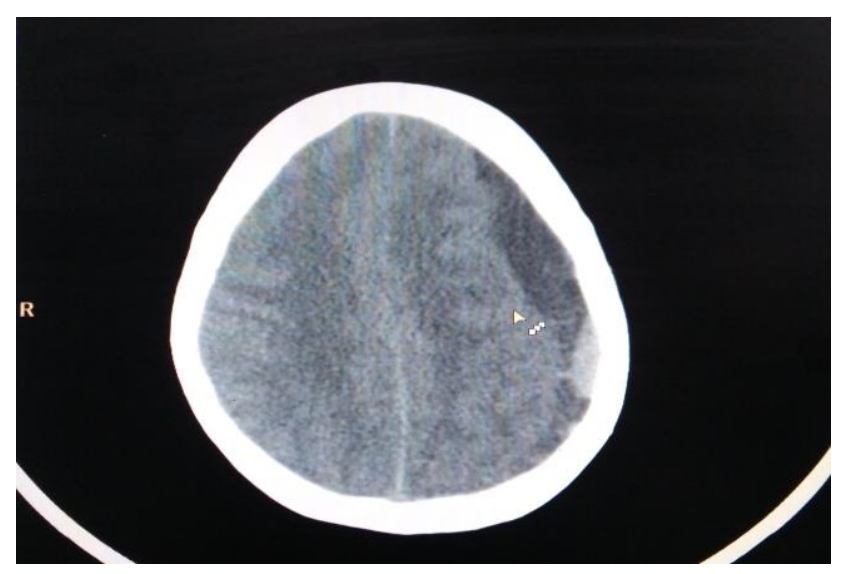

Figure 1: CT scan revealed an acute on chronic SDH left frontotemporo parietal region with midline shift of $7 \mathrm{~mm}$.

Initially managed conservatively with Inj mannitol, analgesics and serial neurological imaging. She later underwent craniotomy and drainage of hematoma for deteriorating neurological status. She had uneventful post op recovery and discharged 4 days after surgery.

\section{Case 2}

30 years old primipara underwent normal delivery with epidural analgesia. Patient had increased blood pressure in antenatal period with normal hematological and biochemical parameters. She was readmitted 02 days after discharge with complaints of severe headache.

There was no history of vomitting, fever and neurological examination revealed no neurological deficit. Initially managed conservatively with no improvement of symptoms. NCCT done was normal and CSF was sent for culture and sensitivity.

Patient developed fever after 02 days of admission. CSF for culture sensitivity grew Streptococcus Pneumoniae sensitive to vancomycin. She was treated for meningitis and patient was started on vancomycin $750 \mathrm{mg}$ twice a day. Patient had complete recovery 02 days after starting antibiotic. Patient was discharged 10 days after admission.

\section{Case 3}

33 years old Primipara, Post IVF twin pregnancy with uneventful antenatal period who underwent normal delivery. Patient was normotensive in antenatal and postnatal period. She was readmitted 08 days after discharge with complaints of excruciating head-ache for 02 days. There was no history of seizures and clinical examination did not reveal any neurological deficit. An NCCT followed by MR venogram was done which revealed left transverse venous sinus thrombosis as shown in (Figure 2).

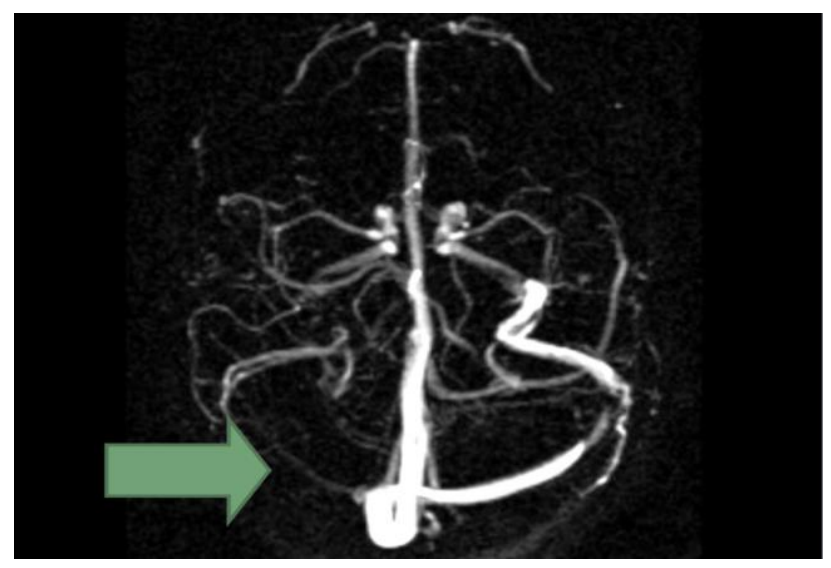

Figure 2: NCCT followed by MR venogram which revealed left transverse venous sinus thrombosis.

She was managed conservatively with inj mannitol, acetaminophen, Tab sodium valproate and inj LMWH in therapeutic doses. Patient had complete resolution of symptoms over the next week and was discharged.

\section{Case 4}

35 years old, primipara who underwent LSCS for failed induction. Patient had increased blood pressure in antenatal period with normal hematological and biochemical parameters.

She had uneventful post-operative period with normal blood pressure records and was discharged on third postoperative day.

Patient was readmitted 04 days after dis-charge with complaints of acute onset headache, vomiting and generalized tonic clonic seizure.

There was no neurological deficit on clinical examination. She underwent MRI which revealed Posterior reversible encephalopathy syndrome (Figure 3). She was man-aged with Inj sodium valproate, mannitol 
and three antihypertensive drugs. She respond-ed well with treatment with complete resolution of symptoms over the next 07 days and was discharged.

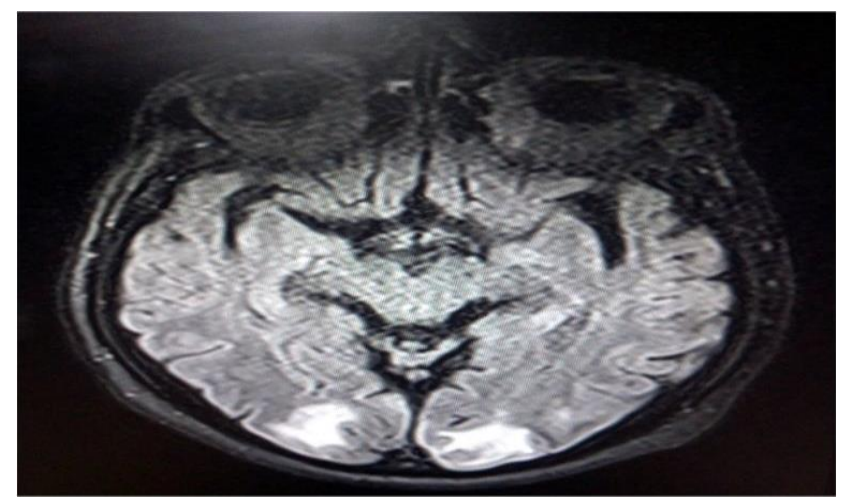

Figure 3: T2 FLAIR image showing PRES.

\section{DISCUSSION}

Postpartum headache is described as a complaint of headache and neck or shoulder pain in the first 6 weeks after delivery. ${ }^{3}$ It is one of the most common symptoms with up to $39 \%$ of parturients experience headache in the first postpartum week. ${ }^{1}$

Tension and migraine headache are major causes of postpartum headache in $47 \%$, pre-eclampsia or eclampsia in $24 \%$ and PDPH in $16 \%$ of patients. ${ }^{1}$ Post dural puncture headache (PDPH) is an iatrogenic complication of neuraxial blockade. The risk of PDPH decreased with small diameter spinal needles but is still seen in many patients undergoing neuraxial blockade. PDPH occurs as early as one day and as late as seven days after dural puncture and lasts 12 hour to seven days. ${ }^{4}$ Other causes of headache like subdural haematoma as in our first case though rare is a possibility and should be excluded by early resort to neuroimaging as was done in our case. Subdural hematoma can sometime develop after dural puncture and is often preceded by symptoms of PDPH. ${ }^{1,2}$ Dural puncture results in leakage of CSF and decreased intracranial pressure (ICP). This re-duction in ICP causes stress on bridging cerebral vessels causing bleeding. There is evidence of increased ICP (e.g., headache, visual disturbances, vomiting, confusion) and focal neurological abnormalities. Patient may present with severe or diffuse headache with an acute or gradual onset, possible focal neurologic deficits and sometimes seizures. History clinical examination and MRI form the mainstay of diagnosis. Hypertension during pregnancy with HELPP is another risk factor of developing intracranial hemorrhage and prompt recognition of intracranial hemorrhages and early neurosurgical intervention is lifesaving. ${ }^{5,6,7}$ Another condition that we must be vigilant of is meningitis which is possible after epidural analgesia that is used for labour analgesia as was in our second case where CSF examination clinched our diagnosis. Postdural puncture meningitis though rare should be on the list of differential diagnosis for any patient who develops headache after dural puncture. It is accompanied by fever, nuchal rigidity, and the presence of Kernig and Brudzinski signs. The diagnosis is confirmed by examination and culture of the CSF. Chemical meningitis, aseptic meningitis, drug related meningitis should also be considered if CSF findings do not suggest bacterial meningitis. ${ }^{8}$

The incidence of cerebral cortical vein thrombosis is increased in pregnancy. Often it is difficult to distinguish cortical vein thrombosis from PDPH because the headache of cortical vein thrombosis aggravates in sitting position. Preceding dural puncture has been reported in several cases. ${ }^{9,10}$ and it has been hypothesized that the reductions in cerebrospinal fluid (CSF) pressure and cerebral vasodilation that accompany dural puncture predispose to thrombosis development. Associated features include focal neurologic signs, seizures, and coma. Cerebral infarction may ensue if diagnosis is delayed. Diagnosis is best confirmed by magnetic resonance imaging. Treatment largely is symptomatic with the aim of preventing seizures. Some studies have suggested that anticoagulation therapy may improve outcome. ${ }^{11}$ Our third case had complaints of excruciating head-ache for 02 days with no seizures or neurological deficit. An NCCT followed by MR venogram confirmed the diagnosis of cortical vein thrombosis. She was symptomatically treated and put on anticoagulation. Another important differential of post-partum headache is Posterior reversible encephalopathy syndrome (PRES) as was in our fourth case. This was first described in 1996 following recognition of a consistent symptom presentation in a diverse group of patients.

Increased blood pressure as in preeclampsia and eclampsia is associated with PRES with pathophysiology similar to hypertensive encephalopathy which causes breach of blood brain barrier integrity. Approximately $25 \%$ of cases of PRES occur in pregnant pa-tients. PRES symptoms include headache, seizures, altered mental status, visual chang-es, and, occasionally, focal neurologic deficits. ${ }^{12}$

Typical signs of PRES are best detected by T2- weighted and fluid-attenuated inversion recovery (FLAIR) MRI which is the gold standard. Typical findings are symmetric edema involving the white matter of the posterior regions of the cerebral hemispheres. Sometimes lesions in the frontal lobes, cerebellum and pons may also be seen. ${ }^{13}$

\section{CONCLUSION}

Headache in postpartum period is taken as a normal finding due to sleep lag, stress and busy obstetricians tend to be oblivious to this symptom in the post-partum period. The aim of bringing out these cases is to highlight the importance of keeping in mind the other causes of headache in postpartum period and to increase vigilance, early resort to neuroimaging and involvement of 
multidisciplinary team in evaluating these cases thorough-ly so as not to miss sinister findings. Just like it is said a stitch in time saves nine, similarly an early diagnosis of cause of headache can prevent serious catastrophic complications.

Funding: No funding sources

Conflict of interest: None declared

Ethical approval: Not required

\section{REFERENCES}

1. Goldszmidt E, Kern R, Chaput A, Macarthur A. The incidence and etiology of postpartum headaches: A prospective cohort study. Can J Anesth. 2005;52(9):971.

2. Stella CL, Jodicke CD, How HY, Harkness UF, Sibai BM. Postpartum headache: is your work-up complete? Am J Obstet Gynecol. 2007;196(4):318e1.

3. Postpartum headache. DH Chestnut, LS Polley, LC Tsen, CA Wong (Eds.), Chest-nut's Obstetric Anesthesia Principles and Practice (4th Ed), Mosby Elsevier, Philadelph-ia (2009), pp. 677-700

4. Choi PT, Galinski SE, Takeuchi L, Lucas S, Tamayo C, Jadad AR. PDPH is a common complication of neuraxial blockade in parturients: a meta-analysis of obstetrical studies. Can J Anesth. 2003;50(5):460-9.

5. Kardash K, Morrow F, Beique F. Seizures after epidural blood patch with undiag-nosed subdural hematoma. Reg Anesth Pain Med. 2002;27(4):433-6.

6. Vau ghan DJ, Stirrup CA, Robinson PN. Cranial subdural haematoma associated with dural puncture in labour. Br J Anaesth. 2000;84(4):518-20.
7. Patabendige M. Acute subdural haemorrhage in the postpartum period as a rare manifestation of possible HELLP (haemolysis, elevated liver enzymes, and low-platelet count) syndrome: a case report. BMC Res. 2014;7(1):408.

8. Baer ET.Post-Dural Puncture Bacterial Meningitis, Anesthesiology 2006; 105(2):381-93.

9. Lockhart EM, Baysinger CL. Intracranial venous thrombosis in the parturient. Anesthe 2007;107(4):652-8.

10. Borum SE, Naul LG, McLeskey CH. Postpartum dural venous sinus thrombosis af-ter postdural puncture headache and epidural blood patch. Anesth. 1997;86(2):487-90.

11. de Bruijn SF, Stam J. Randomized, placebocontrolled trial of antico- agulant treatment with lowmolecular-weight heparin for cerebral sinus thrombosis. Stroke 1999;30(3):484-8.

12. Brewer J, Owens MY, Wallace K, Reeves AA, Morris R, Khan M, et al. Posterior re-versible encephalopathy syndrome in 46 of 47 patients with eclampsia. Am. J. Obstet. Gynecol. 2013;208(6):468.e1-6.

13. Bartynski WS.Posterior reversible encephalopathy syndrome, part 1: fundamental imaging and clinical features.AJNR Am J Neuroradiol. 2008;29(6):103642.

Cite this article as: Bhatt RK, Kumar R, Singh LB. Post partum headache: a case series. Int J Reprod Contracept Obstet Gynecol 2019;8:317-20. 